Meta

Journal des traducteurs

Translators' Journal

\title{
Les retombées terminologiques de l'aide financière
}

\section{Robert Dubuc}

Volume 20, numéro 2, juin 1975

URI : https://id.erudit.org/iderudit/002634ar

DOI : https://doi.org/10.7202/002634ar

Aller au sommaire du numéro

Éditeur(s)

Les Presses de l'Université de Montréal

ISSN

0026-0452 (imprimé)

1492-1421 (numérique)

Découvrir la revue

Citer cet article

Dubuc, R. (1975). Les retombées terminologiques de l'aide financière. Meta, 20(2), 154-157. https://doi.org/10.7202/002634ar d'utilisation que vous pouvez consulter en ligne.

https://apropos.erudit.org/fr/usagers/politique-dutilisation/ 


\section{LES RETOMBÉES TERMINOLOGIQUES DE L'AIDE FINANCIÈRE *}

Dans une société où l'État est appelé à prendre la relève des philanthropes et des mécènes, il n'est pas sans intérêt de comparcr les termes employés en anglais et en français en matière d'aide financière.

A l'échelle des besoins élémentaires, les mots français AIDE et SECOURS s'appliquent à toute somme d'argent versée pour aider quelqu'un à se sortir d'une situation difficile. Ces mots correspondent sensiblement aux mots anglais aid, assistance. Si l'aide s'institutionnalise, il faut parler en français d'ASSISTANCE et en anglais de relief.

L'aide financière peut aussi être accordée en compensation de certains frais qu'une personne doit engager. On parlera alors d'INDEMNITÉ : indemnité de séjour, de déplacement. On rendra dans ce cas «indemnité » par allowance : per diem allowance, travelling allowance. Il ne faut pas confondre «indemnité » et «allocation». L'indemnité est une compensation de frais engagés, tandis que l'ALLOCATION est accordée pour permettre à quelqu'un de subvenir à des besoins particuliers. Les allocations familiales ont ainsi pour objet d'aider la famille à faire face à ses excédents de dépenses. Le mot «allocation » équivaut le plus souvent à l'anglais benefits, quoique l'usage confonde souvent allowance et benefits. Le mot PRESTATION est un quasi-synonyme d'allocation. Il se dit plus proprement des allocations versées par l'État. Ex. : les prestations d'assurancechômage. L'anglais ne fait pas cette distinction. Toutefois, on parlera en anglais de compensation pour désigner une indemnisation en vertu de la loi des accidents du travail. Le français utilise le terme général INDEMNITÉ dans ce sens particulier.

Les secours financiers accordés à des cuvres ou à des organismes peuvent prendre diverses formes. La forme la plus courante est la SUBVENTION : aide financière accordée pour assurer le fonctionnement ou le maintien en existence d'une association ou d'un organisme. Elle peut être versée par l'État, une association ou un groupement. C'est le mot grant qui est employé couramment en anglais en ce sens. Les mots subvention (qui est rare) et subsidy sont des synonymes, mais subsidy s'applique plus particulièrement aux subventions étatiques. Ce mot possède, en outre, un sens spécifique: «A grant of funds or property from a government to a private person or company to assist in the establishment or support of an enterprise deemed advantageous to the public.»Il désigne encore l'argent accordé par un État à un autre État, et enfin il s'applique aux sommes que le gouvernement verse en retour des services qu'on lui rend ou qu'on rend en son nom. Dans tous ces sens, le mot subsidy équivaut à SUBSIDE. L'anglais possède un spécifique sans correspondant exact en français, c'est le terme grantin-aid qui désigne un subside versé par un gouvernement central à un gouvernement local. Ce terme cst d'usage courant au Canada où les niveaux de gouverne-

* I.es définitions qui ont servi de base à cette étude ont été tirées des ouvrages suivants : Webster's Third International Dictionary, 3 vol., Chicago, Encyclopaedia Britannica, 1966. Paul Robert, Dictionnaire alphabétique et analogique de la langue française, 6 vol. et supplément, Paris, Société du Nouveau Littré, 1969

Henri Bénac, Dictionnaire des synonymes. Paris, Hachette, 1973. 
ment sont multiples. C'est encore au terme SUBSIDE qu'il faut recourir pour rendre cette expression, quitte à en étendre quelque peu le sens.

Quand l'aide gouvernementale ne s'applique non plus à des organismes sans but lucratif, mais à des secteurs de l'industrie, c'est le mot bonus qu'il faut employer en anglais. En français, bonus se traduit par ENCOURAGEMENT : aide financière pour permettre l'essor d'une activité, ou par PRIME dont le sens est plus général.

ROBERT DUbUC

\section{RÉSUMÉ TERMINOLOGIQUE}

$\begin{array}{ll}\text { français } & \text { anglais } \\ \text { aide; secours } & \text { aid ; assistance }\end{array}$

Appliqués à un secours financier, ces termes désignent une somme quelconque versée occasionnellement pour permettre à quelqu'un de sortir d'une situation difficile : Aide aux sinistrés du Honduras.

assistance relief

Secours financier versé d'une façon « institutionnalisée ", soit par l'État, soit par un organisme de bienfaisance, pour venir en aide aux personnes dans le besoin. Programme d'assistance aux défavorisés.

indemnité allowance

1. Somme accordée en compensation de certains frais engagés. Indemnité de séjour. compensation benefits

2. Somme accordée pour dédommager des pertes subies à la suite d'un sinistre. Indemnité d'assurance.

allocation

benefits

allowance

Subside accordé pour subvenir à des besoins spéciaux. Allocation familiale.

prestation

benefits

allowance

Subside accordé par l'État pour subvenir à des besoins spéciaux. Prestation d'assurancechômage.

subvention

grant

subsidy

subvention (rare)

Aide financière accordée à titre de secours par l'Êtat, une association ou un groupement. Une subvention du Conseil des arts à un organisme de recherche.

subside subsidy

1. Somme versée par l'État occasionnellement ou régulièrement en rémunération de certains services, sans qu'il y ait nécessairement proportions entre la somme versée et le service rendu : Subside du gouvernement fédéral aux chemins de fer.

2. Aide financière consentie par un État à un autre : Subsides versés par les États-Unis au Vietnam.

grant-in-aid

3. Aide financière d'un gouvernement central accordée à un gouvernement régional : Subsides du gouvernement fédéral aux municipalités.

encouragement; prime

bonus

Aide financière pour permettre l'essor d'une activité : Encouragements accordés à l'industrie laitière. 


\section{QU'EST-CE QUE L'AUDIT ?}

Le terme d'audit recouvrant une réalité anglo-saxonne à l'origine, le problème se pose d'en trouver un équivalent convenable en français. Cette recherche passant nécessairement par la compréhension de la discipline en question, nous fonderons notre brève étude - technique d'abord, terminologique ensuite - sur des documents émanant de la profession elle-même plutôt que de linguistes ou de traducteurs. Nous verrons enfin comment cette notion, comptable au départ, débouche sur le domaine de la gestion.

Essayons d'abord de définir l'audit. D'après un numéro spécial de Hommes et Conmerce ${ }^{1}$ consacré à cette activité, il s'agit d'un examen scientifique et systématique des opérations d'une entreprise qui permet d'émettre un avis sur la régularité et la sincérité de ses états financiers. Le contrôle est scientifique, faisant appel à des techniques mathématiques telles que l'application aux sondages de la théorie des probabilités. 11 est systématique, car tous les postes du bilan et des comptes de résultats sont passés en revue. Par régularité, on entend la conformité aux méthodes et principes comptables généralement admis ainsi qu'aux lois commerciales, fiscales et sociales. La sincérité, enfin, signifie l'expression fidèle de la situation de l'entreprise lors de l'établissement des documents, notamment en ce qui concerne les évaluations extra-comptables (stocks, amortissements, provisions). Axée à l'origine sur la recherche de la fraude et des erreurs, la révision comptable (adoptons provisoirement ce terme) accorde maintenant une importance primordiale au contrôle interne, en raison notamment de la taille croissante des entreprises, de la complexité toujours plus grande de leurs opérations, de l'avènement de l'informatique et... de la hausse des honoraires des réviseurs. Le contrôle peut être complet (full audit), tournant (étalé sur plusieurs exercices) ou faire appel aux sondages. Le réviseur procède par vérifications matérielles (comptages, pointages), recoupements externes et examen de documents. Il peut se faire présenter toutes les pièces qu'il estime utiles à l'exercice de sa fonction. Soumis à un code d'éthique, il doit s'acquitter de sa mission en respectant les diligences normales de la profession.

Nous venons d'employer des termes tels que révision comptable, réviseur, contrôle (interne ou non), soit autant d'équivalents possibles d'audit ou auditor. Il en est d'autres, vérificateur et auditeur par exemple.

Commençons par faire une mise au point au sujet du terme auditeur, dans lequel certains voient, à tort selon nous, un anglicisme. Dérivé du latin auditor, l'auditeur à la Cour des comptes, en France, est celui qui «écoute » les redditions de comptes pour vérifier, apurer, juger la gestion des comptables publics et signaler leurs infractions. Il faudrait donc voir un latinisme (ou un archaïsme) plutôt qu'un anglicisme dans l'usage de ce terme. 11 est cependant permis de lui préfércr d'autres expressions, telles que vérificateur, contrôleur ou réviseur.

1. Revue publiée par l'Association des anciens élèves de l'École des hautes études commerciales de Paris. 
Pourquoi ne pas ajouter à la liste le commissaire aux comptes (ou commissaire de sociétés), dont les fonctions semblent à certains" analogues à celles de l'auditor ? Justement parce que les attributions des commissaires sont plus restreintes que celles de leurs homologues anglo-saxons. Malgré une réforme relativement récente, le commissariat aux comptes suppose un contrôle moins approfondi que l'audit (une preuve en étant que les sociétés françaises désireuses de voir leurs actions cotées à Londres ou à New York doivent faire réviser par des auditors leurs comptes, pourtant déjà examinés par des commissaires). Reprenons donc les trois autres équivalents proposés à la fin du paragraphe précédent.

Le premier, vérificateur, est le plus couramment employé au Canada, dans l'administration publique comme dans le secteur privé. Justifiable techniquement, il présente aussi l'avantage (toujours apprécié du traducteur) de faire partie d'une famille complète (vérifier, vérification, vérificateur). Toutefois, les deux autrcs termes, outre qu'ils offrent la même caractéristique, sont les plus répandus en Europe francophone. Si l'on consulte un annuaire professionnel en France, on y trouve les deux expressions contrôle comptable et révision comptable, celle-ci revenant le plus souvent sous la plume des spécialistes. Ainsi, le président du Conseil supérieur de l'Ordre des experts-comptables français, citant le Robert à l'article révision, rappelle que ce substantif signifie : « examen par lequel on vérifie qu'une chose est bien dans l'état où elle doit être. » La Belgique et la Suisse viennent confirmer la primauté de révision dans l'usage européen : l'annuaire des professions libérales de Bruxelles donne la liste des auditors à la rubrique Réviseurs d'entreprises, tandis que le Code suisse des obligations (à l'article 732) prévoit des mandats de révision. Notons cependant qu'on ne parle pas de réviseur interne, mais de contrôleur (en France) ou de vérificateur (au Canada) interne.

Ce rapide tour d'horizon nous permet moins de conclure à la supériorité de tel ou tel équivalent que de faire une constatation : tant que la loi, dans un souci de protection des actionnaires, des tiers et du grand public, obligera les entreprises à faire examiner leurs comptes par des professionnels indépendants, on pourra rendre audit par révision, contrôle ou vérification (compte tenu des observations formulées au paragraphe précédent). Cependant, les praticiens de cette discipline perçoivent eux-mêmes les limites d'un tel examen, que leur exigence de rigueur porterait dans bien des cas à dépasser pour déboucher sur un véritable contrôle de gestion (management audit). Toutefois, nous tombons là dans un autre domaine, qui mériterait un article à lui seul.

Gilles Gramas

2. Voir la fiche de Radio-Canada sur auditor 